\title{
Cardiac rehabilitation completion is associated with reduced mortality in patients with diabetes and coronary artery disease
}

\author{
Marni J. Armstrong • Ronald J. Sigal • Ross Arena • \\ Trina L. Hauer • Leslie D. Austford • Sandeep Aggarwal • \\ James A. Stone • Billie-Jean Martin
}

Received: 2 September 2014 / Accepted: 23 December 2014 /Published online: 26 January 2015

(C) Springer-Verlag Berlin Heidelberg 2015

\begin{abstract}
Aims Cardiac rehabilitation (CR) reduces the risks of mortality and hospitalisation in patients with coronary artery disease and without diabetes. It is unknown whether patients with diabetes obtain the same benefits from CR.

Methods We retrospectively examined patients referred to a 12 week CR programme between 1996 and 2010. Associations between CR completion vs non-completion and death, hospitalisation rate and cardiac hospitalisation rate were assessed by survival analysis.
\end{abstract}

Electronic supplementary material The online version of this article (doi:10.1007/s00125-015-3491-1) contains peer-reviewed but unedited supplementary material, which is available to authorised users.

M. J. Armstrong $(\bowtie) \cdot$ R. J. Sigal

Cardiovascular and Respiratory Sciences, Cumming School

of Medicine, Diabetes Clinical Trials Unit,

1820 Richmond Road SW, Calgary, AB T2T 5C7, Canada

e-mail: marni.armstrong@ucalgary.ca

R. J. Sigal

Department of Medicine, Cumming School of Medicine,

University of Calgary, Calgary, AB, Canada

R. J. Sigal

Department of Community Health Sciences, Cumming School

of Medicine, University of Calgary, Calgary, AB, Canada

T. L. Hauer $\cdot$ L. D. Austford $\cdot$ S. Aggarwal $\cdot$ J. A. Stone $\cdot$

B.-J. Martin

Department of Cardiac Sciences, Cumming School of Medicine,

University of Calgary, Calgary, AB, Canada

R. Arena $\cdot$ S. Aggarwal $\cdot$ J. A. Stone $\cdot$ B.-J. Martin

TotalCardiology Rehabilitation and Risk Reduction,

Calgary, AB, Canada

R. Arena

Department of Physical Therapy, College of Applied Health

Sciences, University of Illinois at Chicago, Chicago, IL, USA
Results Over the study period, 13,158 participants were referred to $\mathrm{CR}$ (mean $\pm \mathrm{SD}$, age $59.9 \pm 11.1$ years, $28.9 \%$ female, 2,956 [22.5\%] with diabetes). Patients with diabetes were less likely to complete $\mathrm{CR}$ than those without diabetes ( $41 \%$ vs $56 \%, p<0.0001)$. Over a median follow-up of 6.6 years, there were 379 deaths in patients with diabetes vs 941 deaths among those without diabetes ( $12.8 \%$ vs $8.9 \%$ ). Of the non-completers, patients with diabetes had a higher mortality rate compared with those without diabetes $(17.7 \%$ vs $11.3 \%$ ). In patients who completed CR, mortality was lower: $11.1 \%$ in patients with diabetes vs $7.0 \%$ in those without diabetes. In patients with diabetes, CR completion was associated with reduced mortality (HR 0.46 [95\% CI $0.37,0.56]$ ), reduced hospitalisation (HR 0.86 [95\% CI $0.76,0.96]$ ) and reduced cardiac hospitalisation (HR 0.67 [95\% CI 0.54 , 0.84]). The protective associations were similar to those of patients without diabetes. In multivariable adjusted analyses, all of these associations remained significant.

Conclusions Patients with diabetes were less likely to complete $\mathrm{CR}$ than those without diabetes. However, patients with diabetes who completed CR derived similar apparent reductions in mortality and hospitalisation to patients without diabetes.

Keywords Cardiac complications - Cardiac rehabilitation . Diabetes $\cdot$ Exercise $\cdot$ Hospitalisation $\cdot$ Mortality

$\begin{array}{ll}\begin{array}{l}\text { Abbreviations } \\ \text { APPROACH }\end{array} & \begin{array}{l}\text { Alberta Provincial Project for Outcome } \\ \text { Assessment in Coronary Heart disease }\end{array} \\ \text { CAD } & \begin{array}{l}\text { Coronary artery disease } \\ \text { CR }\end{array} \\ \text { Cardiac rehabilitation } \\ \text { IQR } & \text { Interquartile range } \\ \text { MI } & \text { Myocardial infarction }\end{array}$




\section{Introduction}

Cardiac rehabilitation (CR) is recognised as an essential component of comprehensive care for patients with coronary artery disease (CAD) [1]. CR as secondary prevention aims to reduce or prevent morbidity resulting from CAD, reduce subsequent coronary events and deaths, and improve functional status and quality of life [2]. Given the amount and quality of evidence, $\mathrm{CR}$ is recognised as a Class I indication for patients after acute coronary syndrome or coronary revascularisation and for patients with stable angina [1, 3]. Metaanalyses of randomised trials found that exercise-based CR programmes reduced overall mortality by $13 \%$, cardiovascular disease (CVD) related mortality by $26 \%$ and hospital admissions by $31 \%$ compared with patients who received usual care [4]. Large cohort studies of CR have found similar results in patients with CAD [5].

Diabetes is a potent risk factor for CAD [6-8]. It is independently associated with a twofold excess risk of a first myocardial infarction (MI) [9]. The presence of diabetes is also a major risk factor for cardiovascular mortality after acute MI [10-14], with recent data suggesting that patients with diabetes have a twofold increase in 5 year mortality rate [15]. Although patients with diabetes form a large proportion of the CAD population as a whole and of those attending CR programmes [16], it is unclear whether the prognosis of patients with diabetes and CAD is positively influenced by exercise training and behavioural changes after undergoing a CR programme. Adding to the uncertainty, the Look AHEAD trial [17] found that an intensive lifestyle intervention did not reduce the risk of cardiovascular morbidity and mortality among patients with type 2 diabetes.

The objectives of this study were to examine the associations between completion of a CR programme and overall mortality, overall hospitalisations and cardiac hospitalisations in CAD patients with and without diabetes.

\section{Methods}

Study population We examined a prospectively collected database of patients who were referred to outpatient CR with the TotalCardiology Rehabilitation and Risk Reduction programme ("TotalCardiology") between 19 January 1996 and 31 March 2010. Since 1996, the TotalCardiology programme has provided a centralised 12 week multidisciplinary CR programme in Calgary, AB, Canada. All referred patients must have been diagnosed with cardiovascular disease. For all referred patients, the reason for referral, baseline characteristics and provincial health number were recorded regardless of CR programme attendance. Approval for this study was granted by the University of Calgary Conjoint Health Research Ethics Board.
Data sources The Alberta Provincial Project for Outcome Assessment in Coronary Heart disease (APPROACH) database is an ongoing initiative that has prospectively collected detailed clinical information and outcomes on all patients undergoing cardiac catheterisation and/or revascularisation in the Canadian province of Alberta since 1995 [18]. The APPROACH database was used to obtain additional data on patient comorbidities, coronary anatomy, therapeutic interventions (coronary artery bypass grafting or percutaneous coronary intervention) and vital statistics. We also used the Inpatient Discharge Abstract database (an administrative database) to identify hospital readmissions along with visit timing and diagnoses. The TotalCardiology, APPROACH and Inpatient Discharge Abstract databases were linked through the use of provincial health numbers, which are unique to each individual. All participants gave informed consent to be included in the databases.

Patient selection The study population comprised patients with a first referral for CR and a diagnosis of CAD who underwent coronary catheterisation before 31 March 2010. Patients who did not have a valid provincial health number, were less than 18 years of age or who lived outside the Calgary Health Region were excluded. Patients were also excluded if they did not survive for 6 months after catheterisation because such patients would most likely not have the opportunity to be referred to CR. Within this excluded group, only one patient had started CR; he had died within 6 months. Patients identified in the TotalCardiology database who were not found in the APPROACH database (i.e. did not undergo catheterisation) were also excluded because the date of the cohort entry was defined as the date of coronary catheterisation.

Study variables We used the TotalCardiology database to identify those patients who were referred for and completed CR: patients were considered to have completed CR if they completed both the baseline and 12 week post-rehabilitation assessments. Patients who were enrolled for CR but did not complete the programme were classified as non-completers. In previous similar work, our research group found that survival curves were not different between patients who did not enrol and those who enrolled but did not complete the CR programme [5]. Using the unique identifiers, patients identified from the TotalCardiology database were linked to the APPROACH database so that further clinical information could be obtained. Patients were considered to have diabetes if at the time of cardiac catheterisation the patient reported a history of diabetes diagnosed or treated by a physician, or if diabetes was indicated in the patient's hospital chart [19]. In addition, APPROACH clinical data were linked to administrative hospital discharge data to validate the information and diagnoses recorded in the APPROACH database [20]. Data collected on clinical risk factors included age, sex, 
hypertension (physician measured or antihypertensive medication prescribed), smoking status, congestive heart failure, renal disease (defined as a serum creatinine level of $>177 \mu \mathrm{mol} / \mathrm{l}$ ) and a history of MI. The indication and results of coronary catheterisation, including coronary anatomy (summarised by the Duke Jeopardy Score), were also recorded [21]. To ensure that the covariate data reasonably reflected the status of patients at $\mathrm{CR}$, only those who were referred within 1 year of their first catheterisation were included. Mortality data was obtained through the APPROACH database, which includes an ongoing data linkage with the provincial vital statistics data.

Cardiac rehabilitation programme $\mathrm{CR}$ offered through the TotalCardiology programme consisted of a 12 week supervised exercise programme, comprising twice weekly exercise sessions, with participants encouraged to undertake two to three additional weekly sessions on their own. Clinical exercise specialists and registered nurses directly supervised the $1 \mathrm{~h}$ programme sessions to ensure that patients were exercising safely and at an appropriate level of intensity. In these sessions, participants undertook aerobic training (walking on an indoor track or using a treadmill, elliptical trainer or cycle ergometer), including a $5 \mathrm{~min}$ warm-up, 20-60 min steadystate exercise and a $5 \mathrm{~min}$ cool-down. During steady-state exercise, participants exercised at an intensity of $45-85 \%$ of the heart rate reserve, based on the results of a graded exercise test. Following each aerobic training session, a stretching and/or resistance training session with elastic tubing was offered. All patients underwent baseline assessment, including a complete physical examination, anthropometric measurements and a graded exercise test. Changes to medications were made under physician direction, in accordance with the treatment targets set by clinical practice guidelines [22]. In addition, patients were offered classes in stress management, nutrition and smoking cessation, along with referral to a social worker or dietitian, as needed. After completion of the 12 week programme, all patients underwent repeat assessment.

Outcomes All-cause mortality, hospital admission for any cause and cardiac hospitalisation were the outcomes of interest. Outcomes were only considered after the date on which participants attended their first CR session. For those patients who did not attend at least one CR session, outcomes were considered after the median time from catheterisation to CR initiation, which was 84 days. Follow-up was completed on 31 August 2011 to allow a minimum 1 year of follow-up for all patients. Deaths occurring during the entire follow-up period were considered. Cardiac-specific hospitalisations within 1 year of CR completion were determined by the use of International Classification of Diseases, ninth revision, clinical modification (ICD-9-CM; www.icd9data.com/2007/Volume1) codes. The diagnostic codes for MI (ICD-9: 410, 412, 4141; ICD-10:
I21, I22, I2382) and congestive heart failure (ICD-9: 428, 415, 4254, 4298; ICD-10: I50, I255, I420, I429) were employed [23]. Hospitalisation was only considered for patients who entered the study on or after 1 January 1998 because data only became available from that date.

Statistical analyses Differences in baseline clinical characteristics and attendance were assessed according to the presence or absence of diabetes. Categorical variables are presented as frequencies and percentages, and continuous variables as mean \pm SD values. Differences in categorical data were assessed using $\chi^{2}$ tests, and differences in continuous baseline data were assessed using one-way ANOVA. All statistical tests with a two-sided $p$ value of $\leq 0.05$ were considered significant. Cox proportional hazards models were used to determine the association between CR, diabetes status and survival after catheterisation. These models were also utilised to determine the association between CR and freedom from readmission to hospital for both all-cause and cardiac-specific readmissions. These models were adjusted for clinical risk factors obtained from the APPROACH database, including age, sex, smoking status, presence or absence of hypertension, congestive heart failure, chronic obstructive pulmonary disease, dyslipidaemia, peripheral vascular disease, renal disease, liver disease, prior MI, indication for coronary catheterisation, and disease severity according to the Duke Jeopardy Score. Survival time was calculated from the date of the first CR session for those who attended (or the overall median time from cardiac catheterisation to $\mathrm{CR}$ attendance for those who did not attend) to the date on which data were censored or an endpoint occurred. For all-cause and cardiac-specific hospitalisation, data were also censored at the time of death. The proportional hazards assumption was assessed and satisfied for each model by examining the log-log survival curves against time and by comparing Kaplan-Meier and Cox survival curves. The proportional hazards model held for the full follow-up period. Statistical analysis was performed using Stata (version 11; StataCorp, College Station, TX, USA).

\section{Results}

From 19 January 1996 to 31 March 2010, 13,158 patients undergoing cardiac catheterisation were referred to CR. Among these patients, 2,956 (22.5\%) had a diagnosis of diabetes. Baseline demographics and clinical characteristics are shown in Table 1. Patients with diabetes were more likely to have hypertension, congestive heart failure and/or renal failure than those without diabetes (all $p<0.05$ ). Based on their Duke Jeopardy Scores, patients with diabetes were more likely to have high disease severity and left main vessel disease, and less likely to have an ST-elevation MI. A comparison of baseline clinical characteristics of patients who completed vs those 
Table 1 Baseline characteristics of patients referred for CR by diabetes status

\begin{tabular}{lllll}
\hline Characteristic & $\begin{array}{l}\text { Entire cohort } \\
(N=13,158)\end{array}$ & $\begin{array}{l}\text { No diabetes } \\
(n=10,562)\end{array}$ & $\begin{array}{l}\text { Diabetes } \\
(n=2,956)\end{array}$ & $p$ value \\
\hline $\begin{array}{l}\text { Age (years; } \\
\text { mean } \pm \text { SD) }\end{array}$ & $59.9 \pm 11.1$ & $59.6 \pm 11.3$ & $61.1 \pm 10.2$ & $<0.0001$ \\
$\begin{array}{l}\text { Female (\%) } \\
\text { Hypertension (\%) }\end{array}$ & 28.9 & 28.3 & 31.3 & 0.002 \\
Current smoker (\%) & 29.6 & 55.7 & 73.8 & $<0.0001$ \\
Congestive heart & 9.9 & 30.4 & 26.3 & $<0.0001$ \\
$\quad$ failure (\%) & & 8.7 & 14.7 & $<0.0001$ \\
Renal failure (\%) & 1.7 & 1.3 & 3.5 & $<0.0001$ \\
$\begin{array}{l}\text { Prior MI (\%) } \\
\text { Disease severity (Duke Jeopardy Score [\%]) }\end{array}$ & 30.8 & 30.7 & 31.3 & 0.526 \\
$\quad$ Low & 52.0 & 54.2 & 43.1 & \\
$\quad$ High & 31.9 & 29.3 & 42.6 & \\
$\quad$ Left main (\%) & 7.8 & 7.2 & 10.1 & $<0.0001$ \\
STEMI (\%) & 36.6 & 38.0 & 31.0 & $<0.0001$ \\
NSTEMI (\%) & 20.5 & 20.8 & 19.5 & 0.232 \\
\hline
\end{tabular}

STEMI, ST-elevation myocardial infarction; NSTEMI, non-ST-elevation myocardial infarction

${ }^{a}$ Renal failure defined as present if the serum creatinine level is $>177 \mu \mathrm{mol} / \mathrm{l}$

${ }^{\mathrm{b}}$ Left main vessel disease

${ }^{\mathrm{c}}$ Data available on all patients who entered the cohort on or after 1 January 2002

who did not complete $\mathrm{CR}$, with and without diabetes, are shown in electronic supplementary material (ESM) Table 1. Although patients with diabetes had somewhat lower aerobic fitness upon entry to CR, improvements in aerobic fitness over the duration of the CR programme were similar in people with and without diabetes, details of which have been previously reported [24]. Among all those who were referred to the CR programme, 8,582 (65.2\%) initiated the programme and 7,160 (54.4\% of those referred to CR and $83.4 \%$ of those who actually initiated $\mathrm{CR}$ ) completed the CR programme. When stratified by diabetes status, patients with diabetes were less likely than those without diabetes to begin the CR programme $(52.3 \%$ vs $66.6 \%, p<0.001)$. Patients with diabetes were also less likely to complete the 12 week programme $(41.3 \%$ vs $56.2 \%, p<0.001)$. Among those who attended at least one session of the CR programme, $79.0 \%$ of those with diabetes and $84.4 \%$ of those without diabetes completed it $(p<0.001)$. Of the patients who completed $\mathrm{CR}$, data on the number of sessions attended was available for $64 \%$. Participants attended a median of 21 (interquartile range [IQR] 14-29) sessions, and there was no difference in session attendance between patients with and without diabetes $(p=0.93)$. When comparing patients who attended more than the median number of sessions with those who attended fewer, an unadjusted HR of $0.76(95 \%$ CI $0.67,0.87)$ was found, suggesting that those who attended more than the median number of sessions had lower mortality. However, when adjusted for clinical covariates including $\mathrm{CR}$ completion, this association was no longer significant (adjusted HR 1.17 [95\% CI 0.98, 1.39]). Consistent with previously reported results [5], an unadjusted analysis showed an association between the number of sessions attended and mortality (HR 0.98 [95\% CI 0.98, 0.99]). This association was similar in people with and without diabetes; however, when adjusted for clinical covariates this association was no longer significant.

In addition to the two supervised exercise sessions per week, CR participants were asked to complete two to three unsupervised sessions per week. There are limited self-reported data available on the number of exercise sessions completed outside the programme. Overall, patients at their 12 week follow-up visit reported engaging in 3.8 \pm 1.6 sessions per week; this did not differ between patients with diabetes $(3.8 \pm 1.64$ sessions) and those without ( $3.8 \pm 1.61$ sessions; $p=0.953)$.

The median follow-up time was 6.6 years (IQR 3.4-9.4) and there were 1,320 deaths during the follow-up period: 379 in patients with diabetes and 941 in those without diabetes (12.8\% and $8.9 \%$, respectively). Among those who did not complete CR (with diabetes, $n=1,375$; without diabetes, $n=$ 4,623), 244 people with diabetes died and 523 people without diabetes died (17.7\% and $11.3 \%$, respectively). Among patients who did complete $\mathrm{CR}$ (with diabetes, $n=1,221$; without diabetes, $n=5,939$ ), 135 people with diabetes died and 418 people without diabetes died (11.1\% and 7.0\%, respectively). Unadjusted and adjusted HRs for all outcomes are shown in Table 2. In the complete cohort, CR completion was associated with reduced all-cause mortality when compared with noncompletion (adjusted HR 0.55 [95\% CI 0.49, 0.61]; ESM Fig. 1). When patients with diabetes were considered separately (see Fig. 1), the apparent protective association between CR and mortality was at least as strong (adjusted HR 0.46 [95\% CI $0.37,0.58]$ ) as in those without diabetes (adjusted HR 0.59 [95\% CI 0.51, 0.67]). After stratification by indication for initial cardiac catheterisation (MI vs non-MI, stable CAD), patients who underwent cardiac catheterisation for acute MI had a similar improvement in survival following CR. When stratified by diabetes status, the results were also similar (ESM Table 2).

Hospitalisation occurred for 5,127 patients over the study period: 1,113 for patients with diabetes and 4,014 for those without ( $37.7 \%$ and $38.0 \%$, respectively). CR completion was associated with a reduction in all-cause hospitalisation for the entire cohort compared with those who did not complete CR (adjusted HR 0.91 [95\% CI 0.86, 0.96]; ESM Fig. 2a). In stratified analyses (see Fig. 2a), the associations between CR completion and all-cause hospitalisation rates were similar in those with diabetes (adjusted HR 0.90 [95\% CI 0.79, 1.01]) and without diabetes (adjusted HR 0.91 [95\% CI 0.85, 0.97]). In the overall cohort, $\mathrm{CR}$ completion was associated with a 
Table 2 Unadjusted and adjusted Cox proportional HRs for the complete cohort and by diabetes status, comparing CR completers vs non-completers

\begin{tabular}{lllllllll}
\hline Variable & \multicolumn{2}{l}{ Unadjusted HR $(95 \% \mathrm{CI})$} & & \multicolumn{2}{l}{ Adjusted ${ }^{\mathrm{a}} \mathrm{HR}(95 \% \mathrm{CI})$} \\
\cline { 2 - 3 } & Complete cohort & No diabetes & Diabetes & & Complete cohort & No diabetes & Diabetes \\
\hline All-cause mortality & $0.47(0.42,0.53)$ & $0.51(0.45,0.58)$ & $0.46(0.37,0.56)$ & & $0.55(0.49,0.61)$ & $0.59(0.51,0.67)$ & $0.46(0.37,0.58)$ \\
Hospitalisation & $0.87(0.82,0.92)$ & $0.89(0.83,0.94)$ & $0.86(0.76,0.96)$ & & $0.91(0.86,0.96)$ & $0.91(0.85,0.97)$ & $0.90(0.79,1.01)$ \\
Cardiac hospitalisation & $0.71(0.63,0.80)$ & $0.75(0.65,0.86)$ & $0.67(0.54,0.84)$ & & $0.78(0.70,0.88)$ & $0.82(0.71,0.94)$ & $0.71(0.56,0.89)$ \\
\hline
\end{tabular}

${ }^{a}$ Adjusted for baseline age, sex, hypertension, smoking status, congestive heart failure, chronic obstructive pulmonary disease, dyslipidaemia, peripheral vascular disease, renal disease, liver disease, prior MI, indication for coronary catheterisation and disease severity according to the Duke Jeopardy Score

reduction in cardiac-specific hospitalisation rates compared with those who did not complete CR (adjusted HR 0.78 [95\% CI 0.70, 0.88]; ESM Fig. 2b). In stratified analyses (see Fig. 2b), CR had at least as strong a protective effect on cardiac hospitalisation rates in patients with diabetes (adjusted HR $0.71[95 \%$ CI $0.56,0.89])$ as in those without diabetes (adjusted HR 0.82 [95\% CI 0.71, 0.94]).

\section{Discussion}

Completion of CR was associated with significant reductions in mortality, overall hospitalisation and cardiac hospitalisation, which is consistent with previous findings [5]. In patients with diabetes, we found that $\mathrm{CR}$ completion was associated with at least as great a reduction in mortality as in those without diabetes. Similarly, CR completion was associated with a lower rate of cardiac hospitalisation, especially for patients with diabetes. To our knowledge, this cohort of over 13,000 patients (2,956 with diabetes) contains the largest number of CAD

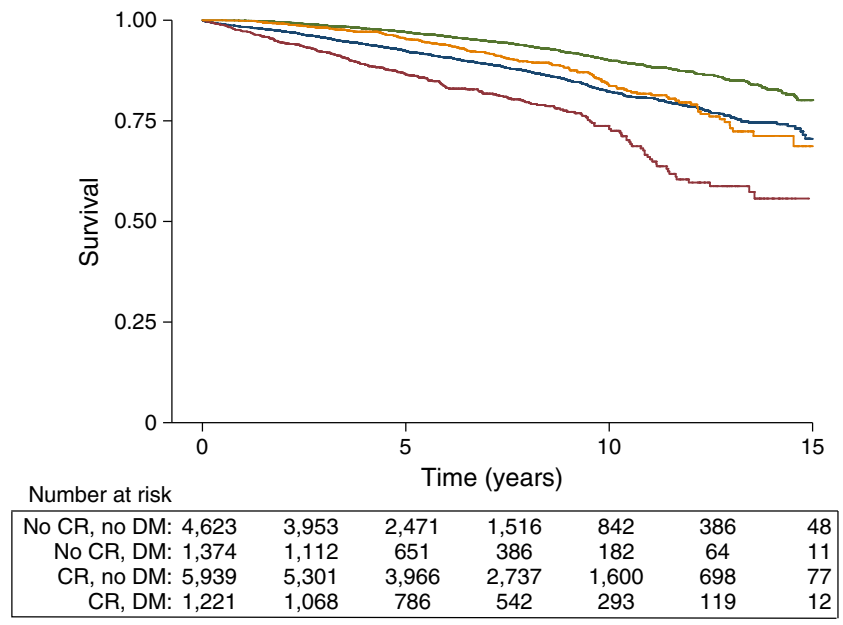

Fig. 1 Adjusted Kaplan-Meier survival curve for patients who completed CR vs those who did not, stratified by diabetes status. Values were adjusted for baseline age, sex, hypertension, smoking status, congestive heart failure, chronic obstructive pulmonary disease, dyslipidaemia, peripheral vascular disease, renal disease, liver disease, prior MI, indication for coronary catheterisation and disease severity according to the Duke Jeopardy Score. Green line: CR, no diabetes mellitus (DM); yellow line: $\mathrm{CR}, \mathrm{DM}$; blue line: no CR, no DM; red line: no CR, DM patients ever to be examined for associations between CR completion and mortality, overall hospitalisation rate or cardiac

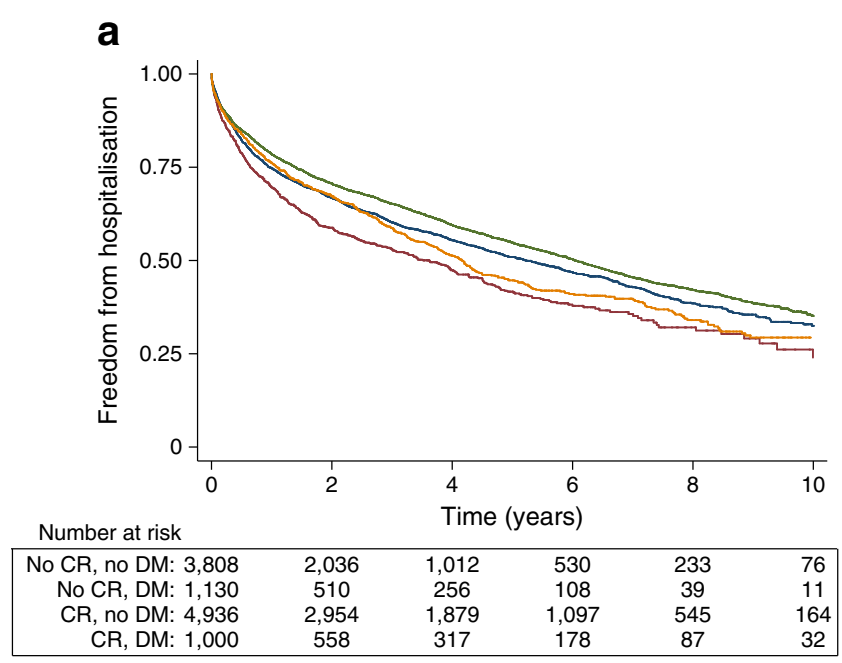

\section{b}

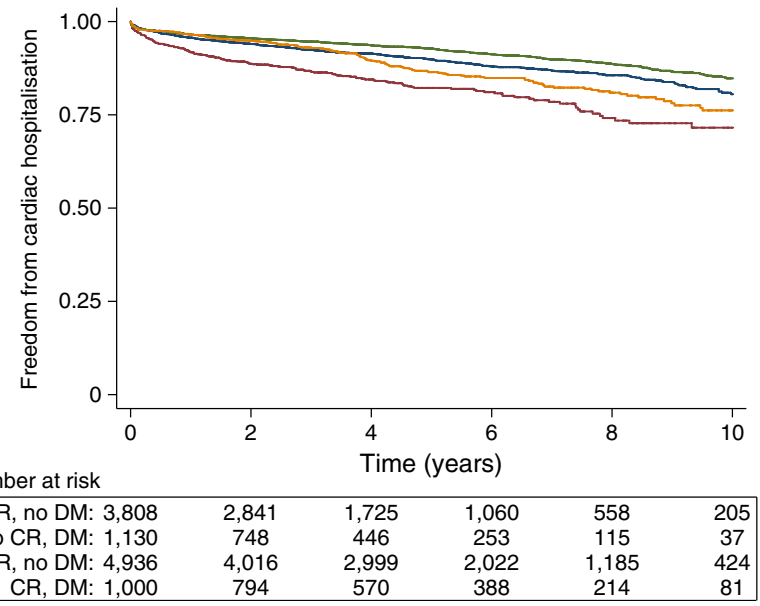

Fig. 2 Adjusted Kaplan-Meier freedom from hospitalisation curves. (a) All-cause hospitalisation stratified by diabetes status. (b) Cardiac hospitalisation stratified by diabetes status. Values were adjusted for baseline age, sex, hypertension, smoking status, congestive heart failure, chronic obstructive pulmonary disease, dyslipidaemia, peripheral vascular disease, renal disease, liver disease, prior MI, indication for coronary catheterisation and disease severity according to the Duke Jeopardy Score. Green line: CR, no diabetes mellitus (DM); yellow line: CR, DM; blue line: no CR, no DM; red line: no CR, DM 
hospitalisation rate, according to diabetes status. These findings suggest that people with diabetes stand to benefit significantly from completing $\mathrm{CR}$, similar to those without diabetes. Therefore, substantial benefits may result from completion of $\mathrm{CR}$, particularly for patients with diabetes. A combination of identifying barriers to $\mathrm{CR}$, targeting adherence interventions and directing resources to patients with diabetes in CR may help to improve post-MI outcomes and has the potential to yield a return on investment.

The apparent reduction in mortality in this cohort is greater than that previously reported in meta-analyses of randomised controlled trials [4] ( $45 \%$ vs $13 \%)$. This may be because the current analysis is not a randomised trial. In addition, those who do not attend CR may have other comorbidities that we were unable to control for in our multivariate model adjustment. The apparent reduction in mortality with $\mathrm{CR}$ also contrasts with the results of the Look AHEAD trial [17]. However, in the Look AHEAD trial, participants randomised to the lifestyle intervention took significantly less cardioprotective medication than those in the control group. Cardioprotective medication is particularly important for secondary prevention, and it is possible that patients in the lifestyle intervention group (and/or their doctors) were excessively confident that lifestyle interventions would reduce the need for medication. In contrast, in our $\mathrm{CR}$ cohort, all patients were strongly encouraged to take cardioprotective medication. We speculate that this difference might account for our apparently different results.

Among the sub-cohort referred for CR, patients with diabetes were less likely to complete the CR programme than those without diabetes $(41.3 \%$ vs $56.2 \%)$. The finding that patients with diabetes are less likely to initiate and adhere to $\mathrm{CR}$ than their non-diabetic counterparts has been observed previously [24-26]. As shown in Table 1, patients with diabetes had higher rates of several comorbidities that may lead to increased dropout for medical reasons. However, in our models adjusted for several comorbidities, the association of CR completion with decreased mortality and hospitalisation rate persisted. Although the data were not readily available, it is possible that rates of depression were higher in patients with diabetes [27], and depression can be an important barrier to completing a supervised exercise programme. Perhaps screening and targeting depression and other motivational issues in the diabetes population may help improve completion rates. Given the substantial benefits that CR can offer to patients with diabetes, efforts should be made to maximise their retention in CR programmes.

We are unaware of other studies comparing mortality in completers and non-completers of CR who are specifically diagnosed with diabetes, although several other studies have examined mortality in CR populations that include participants with and without diabetes. In an evaluation of $418 \mathrm{CR}$ patients, of whom 133 had diabetes, Yu and colleagues [14] found that diabetes independently predicted mortality and acute medical ward admission for cardiac complications. In addition, patients with underlying diabetes had significantly higher mortality compared with those without diabetes $(23.4 \%$ vs $5.5 \%)$. In a larger study of 12,169 men referred for CR, Kavanagh et al [12] found that diabetes was one of the few significant predictors of all-cause death (HR 1.86); others were age, smoking and digoxin use. However, unlike the present study in which the overwhelming majority of participants received guideline-appropriate in-hospital revascularisation and cardioprotective medical therapies, most patients in Kavanagh and colleagues' study (published in 2002) did not undergo revascularisation and received only minimal medical therapy.

Aerobic training, which forms a primary component of CR, is especially beneficial to patients with diabetes. Large cohort studies have demonstrated that in people with type 2 diabetes without $\mathrm{CAD}$, regular physical activity is associated with reductions in cardiovascular and overall mortality of $39-70 \%$, even after adjustment for other CVD risk factors [28-32]. It has also been shown that cardiorespiratory fitness is an independent predictor of mortality in men with type 2 diabetes and that men with low fitness had an adjusted risk of all-cause mortality of 2.1 compared with fit men [33]. Aerobic exercise improves insulin action, blood glucose control, and fat oxidation and storage in the muscle in people with diabetes [34]. We previously reported that improvements in cardiorespiratory fitness were similar in patients with and without diabetes at completion of the 12 week CR programme [24]. However, these changes were not as well maintained in patients with diabetes at the 1 year follow-up. Other studies have reported mixed results, with some reporting similar benefits to patients with and without diabetes $[25,27,35,36]$ and others reporting that patients with diabetes had a less marked overall improvement in exercise capacity [37, 38]. Although aerobic training is probably the most important component of CR, it should be noted that the TotalCardiology programme also involved addressing conventional risk factors such as lipid control, stress management, nutrition and cessation of cigarette smoking.

The strengths of this study include the large population, including a large number of patients with diabetes, prospectively gathered data and linkable clinical databases to obtain a dataset rich in clinical covariates. Another strength is the high completion rate in participants who initiated the CR programme. The completion rates of $84 \%$ in patients without diabetes and $79 \%$ in those with diabetes in the 12 week CR programme are at the high end of the range reported for other CR programmes [39].

Several limitations to the study should be noted. This study is a retrospective analysis of a cohort study rather than a randomised trial, which limits our ability to draw causal inferences. This study is based on observational data, which makes it impossible to be certain that $\mathrm{CR}$ alone was responsible for clinical benefits. Even though we adjusted for many 
comorbidities, it may be that sicker patients were not able to complete CR. Moreover, the data were originally collected for clinical purposes and are therefore subject to imperfect or incomplete data entry.

Given the importance of cardiorespiratory fitness on the prognosis of patients with $\mathrm{CAD}$, it is a limitation that fitness measures were not available for patients who did not attend CR. It is likely that patients who did not attend CR had lower fitness levels, which influenced mortality and hospitalisations. However, a key goal of CR is to improve fitness levels, underscoring the importance of aerobic training as part of secondary prevention. In this cohort of individuals with diabetes, we did not have $\mathrm{HbA}_{1 \mathrm{c}}$ results for most patients and thus could not account for the level of glycaemic control in our multivariate models. In addition, although the models were adjusted for renal disease, information on diabetic complications such as peripheral neuropathy, retinopathy and microalbuminuria was not available for incorporation into multivariate models. The impact of these other microvascular complications in people with diabetes are significant and may extend into other clinically important outcomes (e.g. quality of life) that are not captured using mortality and hospitalisation endpoints.

The definition of diabetes in this dataset could not distinguish between type 1 and type 2 diabetes. However, approximately $90-95 \%$ of reported diabetes in adults is type 2 diabetes [40], so we can presume that the majority of our diabetic patients had type 2 diabetes. The inability to distinguish between type 1 and type 2 diabetes is a limitation of many administrative and clinical databases. Because patients were identified as having diabetes at the time of cardiac catheterisation if they reported a history of diabetes diagnosed or treated by a physician or if diabetes was indicated in the patient hospital chart, we are confident that those classified with diabetes were correctly identified. It is possible that some patients with undiagnosed mild diabetes were misclassified as non-diabetic; such misclassification would bias findings toward the null hypothesis.

\section{Conclusion}

In this analysis of one of the largest cohorts ever studied of patients with $\mathrm{CAD}$, completion of $\mathrm{CR}$ in those with diabetes was associated with significantly lower risks of mortality and cardiac hospitalisation, and this reduction was at least as great as in those without diabetes. However, people with diabetes were less likely to complete $\mathrm{CR}$ after initiating the programme compared with their non-diabetic counterparts. Patients with diabetes comprise a notable proportion of patients in secondary prevention, and it is anticipated that the prevalence of type 2 diabetes in this population will continue to rise. Considering the available evidence on the benefits of $\mathrm{CR}$, it is alarming that
CR is markedly underutilised [39, 41]. Given the impressive clinical (and potential cost-saving) benefits of CR, particularly in those with diabetes, greater emphasis must be placed on improving attendance rates to effective secondary prevention CR programmes after major CVD events.

Acknowledgements The authors would like to thank members of the APPROACH steering committee and staff who helped make this study possible.

Funding MJA was supported by doctoral awards from the Alliance for Canadian Health Outcomes for Research in Diabetes, Alberta InnovatesHealth Solutions and the University of Calgary-Eyes High programme. B-JM was supported by clinical fellowships from Alberta InnovatesHealth Solutions and the Canadian Cardiovascular Outcomes Research Team through the Canadian Institute of Health Research. RJS was supported by a Health Senior Scholar Award from Alberta Innovates-Health Solutions. APPROACH was initially funded with a grant from the W. Garfield Weston Foundation. The ongoing operation of the APPROACH project has been made possible by support from Alberta Health Services, the Libin Cardiovascular Institute of Alberta and the Mazankowski Alberta Heart Institute. The APPROACH initiative has also received contributions from Alberta Health and Wellness and the industry sponsors Merck Frosst Canada Inc., Eli Lilly Canada Inc. and Servier Canada Inc. to support the basic infrastructure of this cardiac registry initiative.

Duality of interest The authors declare that there is no duality of interest associated with this manuscript.

Contribution statement MJA contributed to the conception and design of the project, data analysis and interpretation, and discussions; drafted, reviewed, and edited the manuscript; and approved the final version. B-JM contributed to the conception and design of the project, took the lead in the data analysis, contributed to discussions, reviewed and edited the manuscript, and approved the final version. RJS contributed to the conception and design of the project, contributed to the discussion, drafted, reviewed and edited the manuscript for important intellectual content, and approved the final version. TLH, LDA, RA, JAS and SA contributed to data acquisition, reviewed and revised the manuscript for important intellectual content, and approved the final version. B-JM is the guarantor of this work and, as such, takes responsibility for the integrity of the data and the accuracy of the data analysis.

\section{References}

1. Smith SC, Benjamin EJ, Bonow RO et al (2011) AHA/ACCF secondary prevention and risk reduction therapy for patients with coronary and other atherosclerotic vascular disease: 2011 update: a guideline from the American Heart Association and American College of Cardiology Foundation. Circulation 124:2458-2473

2. Kwan G, Balady GJ (2012) Cardiac rehabilitation 2012: advancing the field through emerging science. Circulation 125:e369-e373

3. Thomas RJ, King M, Lui K, Oldridge N, Pina IL, Spertus J (2010) AACVPR/ACCF/AHA 2010 update: performance measures on cardiac rehabilitation for referral to cardiac rehabilitation/secondary prevention services. J Am Coll Cardiol 56:1159-1167

4. Heran BS, Chen JM, Ebrahim S et al (2011) Exercise-based cardiac rehabilitation for coronary heart disease. Cochrane Database Syst Rev 6, CD001800 
5. Martin BJ, Hauer T, Arena R et al (2012) Cardiac rehabilitation attendance and outcomes in coronary artery disease patients. Circulation 126:677-687

6. Haffner SM, Lehto S, Ronnemaa T, Pyorala K, Laakso M (1998) Mortality from coronary heart disease in subjects with type 2 diabetes and in nondiabetic subjects with and without prior myocardial infarction. N Engl J Med 339:229-234

7. Schramm TK, Gislason GH, Kober L et al (2008) Diabetes patients requiring glucose-lowering therapy and nondiabetics with a prior myocardial infarction carry the same cardiovascular risk: a population study of 3.3 million people. Circulation 117:1945-1954

8. Spencer EA, Pirie KL, Stevens RJ et al (2008) Diabetes and modifiable risk factors for cardiovascular disease: the prospective Million Women Study. Eur J Epidemiol 23:793-799

9. Sarwar N, Gao P, Seshasai SR et al (2010) Diabetes mellitus, fasting blood glucose concentration, and risk of vascular disease: a collaborative meta-analysis of 102 prospective studies. Lancet 375:22152222

10. Behar S, Boyko V, Reicher-Reiss H, Goldbourt U (1997) Ten-year survival after acute myocardial infarction: comparison of patients with and without diabetes. Am Heart J 133:290-296

11. Chyun D, Vaccarino V, Murillo J, Young LH, Krumholz HM (2002) Cardiac outcomes after myocardial infarction in elderly patients with diabetes mellitus. Am J Crit Care 11:504-519

12. Kavanagh T, Mertens DJ, Hamm LF et al (2002) Prediction of longterm prognosis in 12169 men referred for cardiac rehabilitation. Circulation 106:666-671

13. Mak KH, Moliterno DJ, Granger CB et al (1997) Influence of diabetes mellitus on clinical outcome in the thrombolytic era of acute myocardial infarction. GUSTO-I Investigators. Global Utilization of Streptokinase and Tissue Plasminogen Activator for Occluded Coronary Arteries. J Am Coll Cardiol 30:171-179

14. Yu CM, Lau CP, Cheung BM et al (2000) Clinical predictors of morbidity and mortality in patients with myocardial infarction or revascularization who underwent cardiac rehabilitation, and importance of diabetes mellitus and exercise capacity. Am J Cardiol 85:344-349

15. Simpson CR, Buckley BS, McLernon DJ, Sheikh A, Murphy A, Hannaford PC (2011) Five-year prognosis in an incident cohort of people presenting with acute myocardial infarction. PLoS One 6:e26573

16. Lopez-Jimenez F, Kramer VC, Masters B et al (2012) Recommendations for managing patients with diabetes mellitus in cardiopulmonary rehabilitation: an American Association of Cardiovascular and Pulmonary Rehabilitation statement. J Cardiopulm Rehabil Prev 32:101-112

17. LookAHEAD-Research-group (2013) Cardiovascular effects of intensive lifestyle intervention in type 2 diabetes. N Engl J Med 369: 145-154

18. Ghali WA, Knudtson ML (2000) Overview of the Alberta Provincial Project for Outcome Assessment in Coronary Heart Disease. On behalf of the APPROACH investigators. Can J Cardiol 16:1225-1230

19. Graham MM, Ghali WA, Faris PD, Galbraith PD, Norris CM, Knudtson ML (2003) Sex differences in the prognostic importance of diabetes in patients with ischemic heart disease undergoing coronary angiography. Diabetes Care 26:3142-3147

20. Norris CM, Ghali WA, Knudtson ML, Naylor CD, Saunders LD (2000) Dealing with missing data in observational health care outcome analyses. J Clin Epidemiol 53:377-383

21. Califf RM, Phillips HR 3rd, Hindman MC et al (1985) Prognostic value of a coronary artery jeopardy score. J Am Coll Cardiol 5:10551063

22. O'Connor RE, Brady W, Brooks SC et al (2010) Part 10: acute coronary syndromes: 2010 American Heart Association Guidelines for Cardiopulmonary Resuscitation and Emergency Cardiovascular Care. Circulation 122:S787-S817
23. Austin PC, Daly PA, Tu JV (2002) A multicenter study of the coding accuracy of hospital discharge administrative data for patients admitted to cardiac care units in Ontario. Am Heart J 144:290-296

24. Armstrong MJ, Martin BJ, Arena R et al (2014) Patients with diabetes in cardiac rehabilitation: attendance and exercise capacity. Med Sci Sports Exerc 46:845-850

25. Banzer JA, Maguire TE, Kennedy CM, O’Malley CJ, Balady GJ (2004) Results of cardiac rehabilitation in patients with diabetes mellitus. Am J Cardiol 93:81-84

26. Suresh V, Harrison RA, Houghton P, Naqvi N (2001) Standard cardiac rehabilitation is less effective for diabetics. Int J Clin Pract 55: 445-448

27. Milani RV, Lavie CJ (1996) Behavioral differences and effects of cardiac rehabilitation in diabetic patients following cardiac events. Am J Med 100:517-523

28. Hu FB, Stampfer MJ, Solomon C et al (2001) Physical activity and risk for cardiovascular events in diabetic women. Ann Intern Med 134:96-105

29. Hu G, Jousilahti P, Barengo NC, Qiao Q, Lakka TA, Tuomilehto J (2005) Physical activity, cardiovascular risk factors, and mortality among Finnish adults with diabetes. Diabetes Care 28:799-805

30. Gregg EW, Gerzoff RB, Caspersen CJ, Williamson DF, Narayan KMV (2003) Relationship of walking to mortality among US adults with diabetes. Arch Intern Med 163:1440-1447

31. Church TS, Cheng YJ, Earnest CP et al (2004) Exercise capacity and body composition as predictors of mortality among men with diabetes. Diabetes Care 27:83-88

32. Church TS, LaMonte MJ, Barlow CE, Blair SN (2005) Cardiorespiratory fitness and body mass index as predictors of cardiovascular disease mortality among men with diabetes. Arch Intern Med 165:2114-2120

33. Wei M, Gibbons LW (2000) Low cardiorespiratory fitness and physical inactivity as predictors of mortality in men with type 2 diabetes. Ann Intern Med 132:605-611

34. Colberg SR, Albright AL, Blissmer BJ et al (2010) Exercise and type 2 diabetes: American College of Sports Medicine and the American Diabetes Association: joint position statement. Exercise and type 2 diabetes. Med Sci Sports Exerc 42:2282-2303

35. Hindman L, Falko JM, LaLonde M, Snow R, Caulin-Glaser T (2005) Clinical profile and outcomes of diabetic and nondiabetic patients in cardiac rehabilitation. Am Heart J 150:1046-1051

36. Mourot L, Boussuges A, Maunier S et al (2010) Cardiovascular rehabilitation in patients with diabetes. J Cardiopulm Rehabil Prev 30: 157-164

37. St Clair M, Mehta H, Sacrinty M, Johnson D, Robinson K (2014) Effects of cardiac rehabilitation in diabetic patients: both cardiac and noncardiac factors determine improvement in exercise capacity. Clin Cardiol 37:233-238

38. Verges B, Patois-Verges B, Cohen M, Lucas B, Galland-Jos C, Casillas JM (2004) Effects of cardiac rehabilitation on exercise capacity in type 2 diabetic patients with coronary artery disease. Diabet Med 21:889-895

39. Balady GJ, Ades PA, Bittner VA et al (2011) Referral, enrollment, and delivery of cardiac rehabilitation/secondary prevention programs at clinical centers and beyond: a presidential advisory from the American Heart Association. Circulation 124:2951-2960

40. Centre for Disease Control and Prevention National Diabetes Fact Sheet: national estimates and general information on diabetes and prediabetes in the United States, 2011. In U.S. Department of Health and Human Services, Atlanta, GA

41. Arena R, Williams M, Forman DE et al (2012) Increasing referral and participation rates to outpatient cardiac rehabilitation: the valuable role of healthcare professionals in the inpatient and home health settings: a science advisory from the American Heart Association. Circulation 125:1321-1329 